\title{
A database for inborn errors of metabolism in the Indian state of Andhra Pradesh
}

\author{
Shaik Abdul Latheef \\ Department of Biochemistry, School of Life Sciences University of Hyderabad, Hyderabad, India \\ Shaik Abdul Latheef - E-mail: yakheen@gmail.com
}

Received November 18, 2009; Accepted December12, 2009; Published January 21, 2010

\begin{abstract}
:
A Database for Inborn Errors of Metabolism (IEM) in the Indian State of Andhra Pradesh (DIEMISAP), is a continuously updated literature depository containing the extensive information on the regional prevalence and heterogeneity of Inborn Errors of metabolism. We report the construction of a database, a flat file and secondary data resource developed using Microsoft Front Page as the core engine. The database contains 18 summaries regarding 43 IEM disorders reported in the AP population, both in general and region wise, with references and links to the IEM disorder databases available on web and institutes involved in IEM research, in India. Summaries can be accessed from the reference given against an alphabetically arranged list of IEM disorders. The DIEMISAP is a useful user friendly and extendable online resource for information on prevalence of IEM in AP.
\end{abstract}

Availability: http://biochem.uohyd.ernet.in

Keywords: Inborn errors of metabolism, Andhra Pradesh, DIEMISAP, database

Background:

In India, the prevalence of Inborn errors of metabolism (IEM) is one in 2497 newborns and in Andhra Pradesh, one in 1000 newborns[1,2].Due to the improvement in health care and laboratory testing facilities in India, metabolic disorders are increasingly being recognized. Distinct religious communities, hierarchical castes and sub castes and isolated tribal groups inhabiting in the country and high level of endogamy may be responsible for wide variation in the prevalence of IEM in different regions and ethnic groups [3]. There is a perceivable need to develop a database based on region and ethnicity and to develop strategies for the prevention and management of IEM [4, 5]. Andhra Pradesh is the fifth largest state in India, accounting for $8.4 \%$ of territory and has a population of about 76 million. The infant mortality rate is 66 per thousand live births as per 2001 census [6]. The Centre for DNA Finger printing and Diagnostics, has developed a relational database containing records of 15,000 newborns screened for IEM in Hyderabad and accessible to authorized persons in view of sensitive nature of data[7]. To check whether a particular disorder is prevalent in the community or not; and to know the possible management strategy reported, the database providing an instant electronic access to literature on IEM, is of immense help to the physician community. In order to bridge the gap for data access to physician and scientific community on IEM, a database named DIEMISAP has been created.

\section{Methodology:}

Data Sources:

Data for the DIEMISAP are collected from PubMed [8] and Google [9]. Updates for the database take place monthly and the information on the last update is indicated in the home page. The data base contains the literature from 1993 to till date. The impact factor of journals ranged from 0.4 to 4.6. The major contributors of the literature were: Institute of Genetics, Center for DNA Finger Printing and Diagnostics and Center for Cellular and Molecular Biology, Hyderabad.

Database Contents:

The database is constructed to ensure user-friendly environment. Microsoft Office Front Page 2000 is used as the core database engine. Front page provide is a flat filed web enabled database management system. The main menu of the DIEMISAP is indicated on the left of each page, summarizing the database contents and each page is highlighted. The database contains 18 hyperlinked summaries regarding 43 IEM observed in newborn. The list of these IEM disorders are indexed alphabetically and the reference is hyperlinked to PubMed. The database consists of three pages in the menu of home page. In "Andhra Pradesh " page, the sub menu such as "history", "geography", "population", "health infrastructure" and "helath status" are provided to acquaint the user, the first hand information, on the state of Andhra Pradesh, which is the focus of the database. In Prevalence of IEM disorders in AP page, menus like "introduction", Prevalence of IEM disorders in AP" and "region wise prevalence of IEM in AP, are provided.

Prevalence of IEM in AP, both in general and region wise, in percentage and per 1000 subjects, is presented in tabulated form. Hyper homocysteinemia, Alkaptonuria, Generalized aminoaciduria, Hereditary tyrosinemia, Hydroxy kynureninuria, Hyperaminoaciduria, Kynureninuria, Persistent hyperlysinemia, Persistent tyrosinemia, Phenylketonuria, Threoninaemia were more common IEM disorders observed in the state of Andhra Pradesh. Lysosomal storage disorders were found in three sub regions of AP namely Rayalaseema, Telangana and Coastal. IEM disorders like mitochondrial, lipoprotein and amino acid disorders were common in Telangana region. This may be due to the differences in the accessibility of technology, sate-of-art and skilled manpower and possibility of people other than Telangana, approaching nearby areas of other States like Tamil Nadu, Karnataka and Maharashtra. A Comprehensive list of databases on IEM and Institutes involved in IEM research in India, are shown in the page "Electronic Resources and hyperlink to the respective web page is provided, to avail further information.

\section{Database Curation and Maintenance:}

The DIEMISAP is administered locally by Dr. Latheef and his support team at University of Hyderabad. The actual database is hosted on Biochem server (http://biochem.uohyd.ernet.in) (Figure 1), located in the Department of Biochemistry. Data entry and database curation is maintained online through a dedicated IP based and password protected admin module, from which the administrator can access the database contents for modification or update[10]. 


\section{Bioinformation}

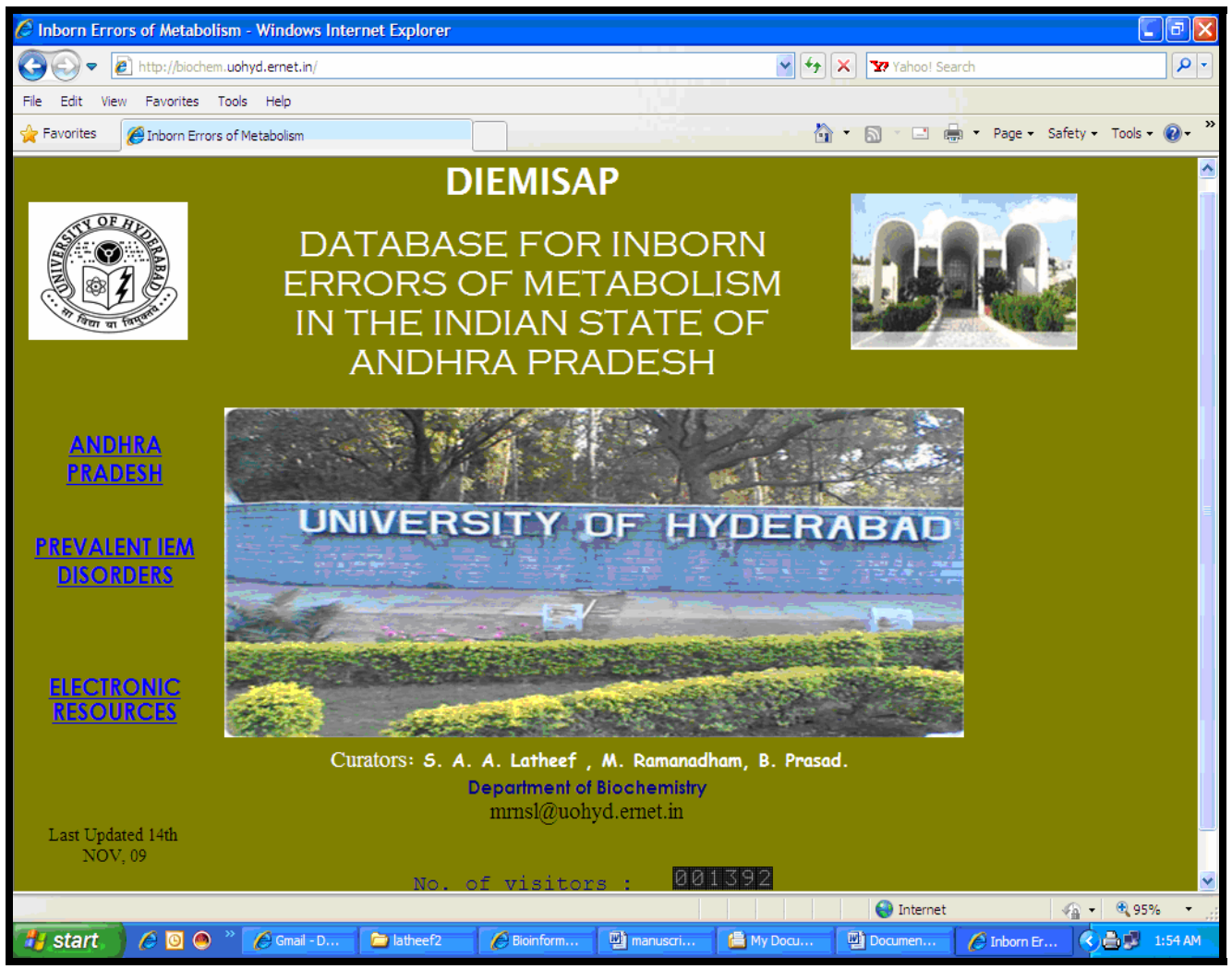

Figure 1: Database screenshot

\section{Database access:}

The DIEMISAP can be accessed on the World Wide Web at the URL address: http://biochem.uohyd.ernet.in. Database access is free-ofcharge for all interested users. This compilation and representation of it are subject to certain copyright policies, especially designed to ensure that this resource remains freely available to all interested persons [10].

\section{Utility:}

This database serves as instant electronic resource and sensitizes the physicians of this region on the prevalence of various IEM in the state. This database may be helpful to the medical students of this region as an educational tool. Policy makers of this State may use the information of the database for making strategy for the prevention and management of prevalent IEM in this region. The information in the database may form the basis for enlisting the disorders for screening programmes in different regions of the state. Researchers of academic and research institutions and industries can make use of the information in the database, for making efforts towards developing cost-effective methods and special diets for the diagnosis and management of IEM. Press and lay people also may use this database for self education and promoting awareness on prevalent IEM in this region. This database may stimulate development of databases on IEM in other regions of India.

\section{Future development:}

We plan to update the database with additional information as and when available. Disadvantages of the DIEMISAP can be considered to be the flat-file platform. If data influx is increased, this can be overcome by shifting to the MySQL based relation database platform[10].

\section{Acknowledgements:}

The author is thankful to UOH-CREBB Center, University of

Hyderabad for providing Post Doctoral Fellowship.

\section{References:}

[1] IC Verma, Indian J Pediatr (2000) 67: 12 [PMID: 11262988].

[2] AR Ramadevi, SM Naushad, Indian J Pediatr (2004) 71: 157 [PMID: 15053381]

[3] Indian Genome Variation Consortium, J Genet (2008) 87: 3 [PMID: 18560169]

[4] AAM Morris, Indian Pediatrics (2000) 37: 1303 [ PMID: 11119331]

[5] KT Shetty, Indian Journal of Clinical Biochemistry (2007) 22: 3.

[6] http://www.aponline.gov.in

[7] http://www.iami.org.in/journal1/nbsdb.asp

[8] http://www.ncbi.nlm.nih.gov/pubmed

[9] http://www.google.co.in

[10] J Zlotogora et al., Human Mutation (2007) 28: 944 [PMID: 17492749]

Edited by P. Kangueane

Citation: Latheef, Bioinformation 4(7): 276-277 (2010)

License statement: This is an open-access article, which permits unrestricted use, distribution, and reproduction in any medium, for noncommercial purposes, provided the original author and source are credited. 